\title{
A THEORY OF FLUCTUATIONS IN PLASMAS
}

\author{
by B. U. FELDERHOF
}

Instituut voor Theoretische Fysica, Rijksuniversiteit, Utrecht

\section{Synopsis}

A theory of thermal fluctuations in plasmas is developed based on a probability ensemble for one-particle distribution functions $f(\boldsymbol{r}, \boldsymbol{v})$. The probability for a specific $f(\boldsymbol{r}, \boldsymbol{v})$ is obtained from the canonical ensemble with the aid of the continuum approximation. Subsequently the probability distribution for deviations from the MaxwellBoltzmann distribution is established. The exponent of this Gaussian distribution is diagonalized by expansion into normal mode solutions of the linearized Vlasov equation. To that purpose van Kampen's normal mode analysis is extended to threedimensional velocity space. Since the development in time of a fluctuation $f_{\mathbf{1}}(r, v)$ follows from the linearized Vlasov equation a general expression can be given for the joint probability distribution of quantities depending linearly on $f_{1}(\boldsymbol{r}, \boldsymbol{v})$ and taken at different points of space and time. By way of example joint probability distributions involving density, potential and electric field, current density and pressure are calculated. The influence of an external force on the fluctuations is investigated. Finally the non-equilibrium case is discussed.

1. Introduction. In recent years there has been considerable interest in the theory of fluctuations in plasmas. Most attention has been paid to the fluctuations in density, because these are directly related to the problem of scattering of electromagnetic waves. In this connection the well-known Bowles' experiment on radar back-scattering from the ionosphere should be mentioned ${ }^{1}$ ). The theory was at first restricted to thermal equilibrium plasmas $\left.{ }^{2}{ }^{3}\right)^{4}$ ) but has later been extended to the non-equilibrium case $\left.\left.{ }^{5}\right)^{6}\right)^{7}$ ).

For thermal equilibrium some of the early results were derived from the Nyquist noise theorem $\left.{ }^{2}\right)^{8}$ ). The conductivity used here was obtained from linearized Vlasov theory; recently an attempt has been made to include collisions $\left.{ }^{9}\right)$. Two aequivalent methods have been proposed which also cover the non-equilibrium case. The first one is based on the concept of independent dressed particles ${ }^{7}{ }^{10}$ ). The second method uses two-time two-particle distribution functions for which explicit expressions are obtained in suitable approximation from the Liouville hierarchy $\left.{ }^{5}\right)^{6}$ ).

The present paper is for the most part devoted to thermal equilibrium fluctuations. An independent method is expounded by which the familiar results for the density fluctuations, microfield spectrum and correlations 
in potential are recovered. We also present some results concerning current density and pressure fluctuations which to our knowledge are new, but which can equally well be obtained by other methods. We have restricted ourselves to the electron gas with positive background, but the treatment can without difficulty be extended to many-component plasmas.

The basic probability distribution of the fluctuations is obtained from the canonical ensemble of equilibrium statistical mechanics, treated in the continuum approximation. In earlier applications this approximation consisted of a division of ordinary space into cells containing a large number of particles $\left.\left.{ }^{11}\right)^{12}\right)^{13}$ ). The canonical ensemble yields the probability for a specific distribution of the particles over the cells, i.e. the probability for a specific density function $n(\boldsymbol{r})$; the latter probability then serves as a basis for further calculations. In our method the continuum approximation is extended to six dimensions; instead of ordinary space the position-velocity or $\mu$-space is divided into cells. The canonical ensemble then yields the probability for a specific distribution of particles over these cells, i.e. the probability for a specific one-particle distribution function $f(\boldsymbol{r}, \boldsymbol{v})$.

The great advantage of this approach is that the development in time of the fluctuations can be followed. Unlike the density, the distribution function evolves in time according to an equation containing only $f(\boldsymbol{r}, \boldsymbol{v})$ itself. In the continuum approximation it is natural to take for this equation the Vlasov equation, although in principle one could also take collisions into account by using a Boltzmann or Fokker-Planck equation. We shall however neglect collisions; moreover, since we are dealing with fluctuations we may restrict ourselves to the Vlasov equation in its linearized form.

In sections 2 and 3 the basic probability ensemble for fluctuations $f_{1}(\boldsymbol{r}, \boldsymbol{v})$ in the distribution function is established. This ensemble is Gaussian and it appears that the exponent which is quadratic in $f_{1}(\boldsymbol{r}, \boldsymbol{v})$ is diagonalized when $f_{1}(\boldsymbol{r}, \boldsymbol{v})$ is expanded into normal mode solutions of the linearized Vlasov equation. In sections 4 to 8 a normal mode analysis is presented which is an extension of the work of van Kampen ${ }^{14}$ ) and Case ${ }^{15}$ ) to three-dimensional velocity space.

In section 9 the diagonalization is performed and section 10 contains a general expression for the joint probability distribution of quantities depending linearly on $f_{1}(\boldsymbol{r}, \boldsymbol{v})$. In sections 11 to 14 the general result is applied to fluctuations in density, potential, electric field, current density and pressure. Sections 15 and 16 deal with the influence of an external force on the fluctuations and section 17 treats the test particle problem as an example. In the last section the non-equilibrium case is discussed.

2. Probability distribution for 1-particle distribution functions. We shall consider a gas of $\mathrm{N}$ electrons of charge $e$, mass $m$ in a voliume $\Omega$ with a positive neutralizing background of charge density $-n_{0} e$, where $n_{0}=N / \Omega$. 
The electrons interact via the Coulomb-interaction

$$
w\left(\boldsymbol{r}, \boldsymbol{r}^{\prime}\right)=\frac{e^{2}}{\left|\boldsymbol{r}-\boldsymbol{r}^{\prime}\right|} .
$$

The probability that the electrons are distributed in a specific fashion over the six-dimensional $(\boldsymbol{r}, \boldsymbol{v})$-space is determined by the canonical ensemble and will be obtained explicitly by use of the continuum approximation. This approximation can be extended to six dimensions in the following way. Divide $\mu$-space into cells of volume $\Delta$ centered at points $\boldsymbol{R}_{i}, \boldsymbol{V}_{i}$; the cells should be large enough to contain many particles and so small that the kinetic energy $\frac{1}{2} m v^{2}$ and the interaction $w\left(\boldsymbol{r}, \boldsymbol{r}^{\prime}\right)$ do not vary appreciably over the cells. Let $F_{i}$ denote the number of particles in the $i$-th cell. A configuration is defined by the set of numbers $\left\{F_{i}\right\}$. The total number of particles of a given configuration is

$$
\sum_{i} F_{i}=N
$$

and its energy is

$$
\begin{aligned}
\frac{1}{2} m \sum_{i} V_{i}^{2} F_{i}+\frac{1}{2} \sum_{i, j} w\left(\boldsymbol{R}_{i}, \boldsymbol{R}_{j}\right) F_{i} F_{j}-\sum_{i, k} w\left(\boldsymbol{R}_{i},\right. & \left.\boldsymbol{R}_{k}\right) F_{i} N_{0}+ \\
& +\frac{1}{2} \sum_{k, l} w\left(\boldsymbol{R}_{k}, \boldsymbol{R}_{l}\right) N_{0}^{2} .
\end{aligned}
$$

The sums over $k$ and $l$ run over the corresponding cells in ordinary space of volume $d$ and $N_{0}=n_{0} d$. In the terms with $\boldsymbol{R}=\boldsymbol{R}^{\prime}$ the interaction should be replaced by an average of $w\left(\boldsymbol{r}, \boldsymbol{r}^{\prime}\right)$ over the space cell in order to avoid divergencies. The last sum derives from the interaction of the background with itself and serves to cancel the second and third sum when the electrons are distributed homogeneously over space. The canonical ensemble yields for the probability of a specific configuration

$$
\begin{aligned}
P\left\{F_{i}\right\}=\frac{N !}{\prod_{i} F_{i} !} \exp \left[-\frac{1}{2} \beta m \sum_{i}\right. & V_{i}^{2} F_{i}-\frac{1}{2} \beta \sum_{i, j} w_{i j} F_{i} F_{j}+ \\
& \left.+\beta \sum_{i, k} w_{i k} F_{i} N_{0}-\frac{1}{2} \beta \sum_{k, l} w_{k l} N_{0}^{2}\right] / \sum_{\left\{F_{i}\right\}} P,
\end{aligned}
$$

where $\beta=1 / k_{B} T$. In Stirling's approximation

$$
\begin{array}{r}
P\left\{F_{i}\right\}=\exp \left[\sum_{i}\left(F_{i}-F_{i} \log F_{i}\right)\right. \\
-\frac{1}{2} \beta m \sum_{i} V_{i}^{2} F_{i}-\frac{1}{2} \beta \sum_{i, j} w_{i j} F_{i} F_{j}+ \\
\left.+\beta \sum_{i, k} w_{i k} F_{i} N_{0}-\frac{1}{2} \beta \sum_{k, l} w_{k l} N_{0}^{2}\right] / \sum_{\left\{F_{i}\right\}} P .
\end{array}
$$

Hence one obtains by use of $F_{i}=f\left(\boldsymbol{R}_{i}, \boldsymbol{V}_{\boldsymbol{i}}\right) \Delta$ and transforming sums into integrals, for the probability of a specific one-particle distribution function

$$
P\{f(\boldsymbol{r}, \boldsymbol{v})\}=\exp \Psi\{f(\boldsymbol{r}, \boldsymbol{v})\} / \int P,
$$


where

$$
\begin{aligned}
\Psi\{f(\boldsymbol{r}, \boldsymbol{v})\} & =\int\left[f(\boldsymbol{r}, \boldsymbol{v})-f(\boldsymbol{r}, \boldsymbol{v}) \log f(\boldsymbol{r}, \boldsymbol{v})-\frac{1}{2} \beta m v^{2} f(\boldsymbol{r}, \boldsymbol{v})\right] \mathrm{d} \boldsymbol{r} \mathrm{d} \boldsymbol{v}- \\
& -\frac{1}{2} \beta \int w\left(\boldsymbol{r}, \boldsymbol{r}^{\prime}\right) f(\boldsymbol{r}, \boldsymbol{v}) f\left(\boldsymbol{r}^{\prime}, \boldsymbol{v}^{\prime}\right) \mathrm{d} \boldsymbol{r} \mathrm{d} \boldsymbol{v} \mathrm{d} \boldsymbol{r}^{\prime} \mathrm{d} \boldsymbol{v}^{\prime}+ \\
& +\beta \int w\left(\boldsymbol{r}, \boldsymbol{r}^{\prime}\right) f(\boldsymbol{r}, \boldsymbol{v}) n_{0} \mathrm{~d} \boldsymbol{r} \mathrm{d} \boldsymbol{v} \mathrm{d} \boldsymbol{r}^{\prime}-\frac{1}{2} \beta \int w\left(\boldsymbol{r}, \boldsymbol{r}^{\prime}\right) n_{0}^{2} \mathrm{~d} \boldsymbol{r} \mathrm{d} \boldsymbol{r}^{\prime} .
\end{aligned}
$$

3. Fluctuations in the distribution function. It will be shown that the probability distribution (2.5) has a sharp maximum when $f(\boldsymbol{r}, \boldsymbol{v})$ is the thermal equilibrium distribution and we shall consider fluctuations around this maximum. In order to find the maximum the first functional derivative of $\Psi$ is taken

$$
\begin{array}{r}
\frac{\delta \Psi}{\delta f(\boldsymbol{r}, \boldsymbol{v})}=-\log f(\boldsymbol{r}, \boldsymbol{v})-\frac{1}{2} \beta m v^{2}-\beta \int w\left(\boldsymbol{r}, \boldsymbol{r}^{\prime}\right) \\
f\left(\boldsymbol{r}^{\prime}, \boldsymbol{v}^{\prime}\right) \mathrm{d} \boldsymbol{r}^{\prime} \mathrm{d} \boldsymbol{v}^{\prime}+ \\
+\beta \int w\left(\boldsymbol{r}, \boldsymbol{r}^{\prime}\right) n_{0} \mathrm{~d} \boldsymbol{r}^{\prime} .
\end{array}
$$

Since $\Psi$ must be maximized subject to the condition

$$
\int f(\boldsymbol{r}, \boldsymbol{v}) \mathrm{d} \boldsymbol{r} \mathrm{d} \boldsymbol{v}=N,
$$

one finds that $\Psi$ is stationary for

$-\log f(\boldsymbol{r}, \boldsymbol{v})-\frac{1}{2} \beta m v^{2}-\beta \int w\left(\boldsymbol{r}, \boldsymbol{r}^{\prime}\right) f\left(\boldsymbol{r}^{\prime}, \boldsymbol{v}^{\prime}\right) \mathrm{d} \boldsymbol{r}^{\prime} \mathrm{d} \boldsymbol{v}^{\prime}+\beta \int w\left(\boldsymbol{r}, \boldsymbol{r}^{\prime}\right) n_{0} \mathrm{~d} \boldsymbol{r}^{\prime}-\alpha=0$.

The Lagrange parameter $\alpha$ is to be chosen such that the normalization condition (3.2) is fulfilled. These equations are surely satisfied by the thermal equilibrium distribution

$$
f_{e q}(\boldsymbol{r}, \boldsymbol{v})=n_{0} f_{M B}(\boldsymbol{v})=n_{0}\left(\frac{\beta m}{2 \pi}\right)^{\frac{3}{2}} \exp \left[-\frac{1}{2} \beta m v^{2}\right]:
$$

In order to show that $P$ has a maximum for this $f$ we take the second functional derivative of $\Psi$

$$
\frac{\delta^{2 \Psi}}{\delta f(\boldsymbol{r}, \boldsymbol{v}) \delta f\left(\boldsymbol{r}^{\prime}, \boldsymbol{v}^{\prime}\right)}=-\frac{\delta\left(\boldsymbol{r}-\boldsymbol{r}^{\prime}\right) \delta\left(\boldsymbol{v}-\boldsymbol{v}^{\prime}\right)}{f(\boldsymbol{r}, \boldsymbol{v})}-\beta w\left(\boldsymbol{r}, \boldsymbol{r}^{\prime}\right),
$$

which is easily shown to be negative definite. This establishes at the same time that the maximum is the only stationary point.

Finally one finds

$$
\begin{aligned}
& \Psi\left\{n_{0} f_{M B}(\boldsymbol{v})+f_{1}(\boldsymbol{r}, \boldsymbol{v})\right\}-\Psi\left\{n_{0} f_{M B}(\boldsymbol{v})\right\}= \\
& =-\frac{1}{2} \int \frac{f_{1}^{2}(\boldsymbol{r}, \boldsymbol{v})}{n_{0} f_{M B}(\boldsymbol{v})} \mathrm{d} \boldsymbol{r} \mathrm{d} \boldsymbol{v}-\frac{\beta}{2} \int w\left(\boldsymbol{r}, \boldsymbol{r}^{\prime}\right) f_{1}(\boldsymbol{r}, \boldsymbol{v}) f_{1}\left(\boldsymbol{r}^{\prime}, \boldsymbol{v}^{\prime}\right) \mathrm{d} \boldsymbol{r} \mathrm{d} \boldsymbol{v} \mathrm{d} \boldsymbol{r}^{\prime} \mathrm{d} \boldsymbol{v}^{\prime}
\end{aligned}
$$

to second order in $f_{1}(\boldsymbol{r}, \boldsymbol{v})$, which yields for the probability distribution of 
the fluctuations

$$
\begin{aligned}
P\left\{f_{1}(\boldsymbol{r}, \boldsymbol{v})\right\}=\exp [ & -\frac{1}{2} \int \frac{f_{1}^{2}(\boldsymbol{r}, \boldsymbol{v})}{n_{0} f_{M B}(\boldsymbol{v})} \mathrm{d} \boldsymbol{r} \mathrm{d} \boldsymbol{v}- \\
& \left.\quad-\frac{\beta e^{2}}{2} \int \frac{f_{1}(\boldsymbol{r}, \boldsymbol{v}) f_{1}\left(\boldsymbol{r}^{\prime}, \boldsymbol{v}^{\prime}\right)}{\left|\boldsymbol{r}-\boldsymbol{r}^{\prime}\right|} \mathrm{d} \boldsymbol{r} \mathrm{d} \boldsymbol{v} \mathrm{d} \boldsymbol{r}^{\prime} \mathrm{d} \boldsymbol{v}^{\prime}\right] / \int P .
\end{aligned}
$$

This probability ensemble enables one to calculate the probability distribution of any quantity which can be expressed in terms of $f_{1}(\boldsymbol{r}, \boldsymbol{v})$, or the joint distribution of pairs of such quantities, etc.. One can, however, go further: It is also possible to calculate joint probabilities of quantities taken at different times, because in the continuum approximation the development in time of a fluctuation $f_{1}(\boldsymbol{r}, \boldsymbol{v})$ is governed by the linearized Vlasov equation.

In order to perform such calculations explicitly it is appropriate to expand $f_{1}(\boldsymbol{r}, \boldsymbol{v})$ into normal mode solutions of the linearized Vlasov equation. It will be shown subsequently that by this expansion the quadratic expression in the exponent of (3.7) is diagonalized. The next few sections are devoted to a normal mode analysis of the linearized Vlasov equation.

4. Van Kampen normal modes. The linearized Vlasov equation for $f_{1}(\boldsymbol{r}, \boldsymbol{v})$ reads

$$
\frac{\partial f_{1}}{\partial t}+\boldsymbol{v} \cdot \frac{\partial f_{1}}{\partial \boldsymbol{r}}+\frac{n_{0} e^{2}}{m} \frac{\partial f_{0}}{\partial \boldsymbol{v}} \cdot \int \frac{\boldsymbol{r}-\boldsymbol{r}^{\prime}}{\left|\boldsymbol{r}-\boldsymbol{r}^{\prime}\right|^{3}} f_{1}\left(\boldsymbol{r}^{\prime}, \boldsymbol{v}^{\prime}, t\right) \mathrm{d} \boldsymbol{r}^{\prime} \mathrm{d} \boldsymbol{v}^{\prime}=0 .
$$

Here $n_{0} f_{0}(v)$ is any spatially homogeneous distribution; in view of the discussion in the last section we do not yet specialize to the MaxwellBoltzmann distribution, but we shall assume that $f_{0}(v)$ is stable in the sense that (4.1) has no solutions exponentially growing or decreasing in time.

By Fourier analysis in space

$$
f_{1}(\boldsymbol{r}, \boldsymbol{v}, t)=\int f_{\boldsymbol{k}}(\boldsymbol{v}, t) \mathrm{e}^{i \boldsymbol{k} \cdot \boldsymbol{r}} \mathrm{d} \boldsymbol{k}
$$

one obtains from (4.1)

$$
\frac{\partial f_{k}}{\partial t}+i \boldsymbol{k} \cdot \boldsymbol{v} f_{k}-i \frac{\omega_{p}^{2}}{k^{2}} \boldsymbol{k} \cdot \frac{\partial f_{0}}{\partial \boldsymbol{v}} \int f_{\boldsymbol{k}}\left(\boldsymbol{v}^{\prime}, t\right) \mathrm{d} \boldsymbol{v}^{\prime}=0
$$

where $\omega_{p}^{2}=4 \pi n_{0} e^{2} / m$ is the plasma frequency. This equation can be simplified by integration over the two velocity components perpendicular to $\boldsymbol{k}$. For fixed $\boldsymbol{k}$ we shall always take the $z$-axis in the direction of $\boldsymbol{k}$. Defining

one obtains

$$
f_{z}\left(v_{z}, t\right)=\int f_{k}(v, t) \mathrm{d} v_{x} \mathrm{~d} v_{y}
$$

$$
\frac{\partial f_{z}}{\partial t}+i k v_{z} f_{z}-i k u_{p}^{2} F^{\prime}\left(\boldsymbol{k}, v_{z}\right) \int f_{z}\left(v_{z}^{\prime}, t\right) \mathrm{d} v_{z}^{\prime}=0
$$


where $u_{p}^{2}=\omega_{p}^{2} / k^{2}$ and $F^{\prime}(\boldsymbol{k}, u)$ is the derivative with respect to $u$ of

$$
F(\boldsymbol{k}, u)=\int f_{0}(\boldsymbol{v}) \delta\left(u-\frac{\boldsymbol{k} \cdot \boldsymbol{v}}{k}\right) \mathrm{d} \boldsymbol{v} .
$$

This function clearly depends only on the direction of $\boldsymbol{k}$.

Equation (4.5) has been studied in normal mode analysis by van $\mathrm{Kampen}{ }^{14}$ ) and $\mathrm{Case}^{15}$ ). One looks for stationary solutions of the form

$$
f_{z}\left(v_{z}, t\right)=g_{\boldsymbol{k}, u}\left(v_{z}\right) \mathrm{e}^{-\mathrm{i} \omega t},
$$

where $u$ stands for $\omega / k$. It follows from (4.5) that $g_{\boldsymbol{k}, u}\left(v_{z}\right)$ must satisfy

$$
\left(u-v_{z}\right) g_{\boldsymbol{k}, u}\left(v_{z}\right)+u u_{\nu}^{2} F^{\prime}\left(\boldsymbol{k}, v_{z}\right) \int g_{\boldsymbol{k}, u}\left(v_{z}^{\prime}\right) \mathrm{d} v_{z}^{\prime}=0 .
$$

One finds as normal modes

$$
g_{\boldsymbol{k}, u}\left(v_{z}\right)=\frac{P}{\pi} \frac{Y\left(\boldsymbol{k}, v_{z}\right)}{u-v_{z}}+X(\boldsymbol{k}, u) \delta\left(u-v_{z}\right),
$$

where $P$ stands for principal value, and where $X$ and $Y$ are the real and imaginary part of*)

$$
Z(\boldsymbol{k}, u)=1-u_{p}^{2} \int \frac{F^{\prime}\left(\boldsymbol{k}, v_{z}\right)}{v_{z}-u-i \varepsilon} \mathrm{d} v_{z},
$$

so that

$$
\begin{aligned}
& X(\boldsymbol{k}, u)=1-u_{\mu}^{2} P \int \frac{F^{\prime}\left(\boldsymbol{k}, v_{z}\right)}{v_{z}-u} \mathrm{~d} v_{z}, \\
& . \quad Y(\boldsymbol{k}, u)=-\pi u_{p}^{2} F^{\prime}(\boldsymbol{k}, u) .
\end{aligned}
$$

The solution (4.9) is normalized to unity. There is a normal mode for every real value of $u$ and together these solutions constitute a complete set, i.e. any solution of (4.5) can be written in the form

$$
f_{z}\left(v_{z}, t\right)=\int C_{0}(\boldsymbol{k}, u) g_{\boldsymbol{k}, u}\left(v_{z}\right) \mathrm{e}^{-i k u t} \mathrm{~d} u .
$$

The coefficients $C_{0}(\boldsymbol{k}, u)$ can be found from $g_{z}\left(v_{z}, t\right)$ at a particular instant of time, e.g. $t=0$, by solving a singular integral equation.

5. Normal modes in 3-dimensional velocity space. The van Kampen normal modes do not suffice for our purpose since we must be able to expand functions of the form $f_{1}(\boldsymbol{r}, \boldsymbol{v})$. One can however extend the analysis to three dimensions in velocity space by looking for stationary solutions of (4.3) of the form

$$
f_{k}(\boldsymbol{v}, t)=g_{\boldsymbol{k}, u}(\boldsymbol{v}) \mathrm{e}^{-i \omega t} .
$$

*) The function $Z(\boldsymbol{k}, \omega / k)$ is often denoted by $\varepsilon(\boldsymbol{k}, \omega)$. 
It follows from (4.3) that $g_{k, u}(\boldsymbol{v})$ must satisfy

$$
\left(u-v_{z}\right) g_{\boldsymbol{k}, u}(\boldsymbol{v})+u_{\nu}^{2} \frac{\partial f_{0}}{\partial v_{z}} \int g_{\boldsymbol{k}, u}\left(\boldsymbol{v}^{\prime}\right) \mathrm{d} \boldsymbol{v}^{\prime}=0 .
$$

Clearly this equation must admit solutions which reduce to (4.9) when one integrates over $v_{x}$ and $v_{y}$. Such solutions are

$$
g_{\boldsymbol{k}, u}^{0}(\boldsymbol{v})=\frac{\partial f_{0} / \partial v_{z}}{F^{\prime}\left(\boldsymbol{k}, v_{z}\right)}\left[\frac{P}{\pi} \frac{Y\left(\boldsymbol{k}, v_{z}\right)}{u-v_{z}}+X(\boldsymbol{k}, u) \delta\left(u-v_{z}\right)\right] .
$$

Obviously, this expression only makes sense for values of $u$ for which $F^{\prime}(\boldsymbol{k}, u)$ does not vanish, but we shall henceforth make the simplifying and unessential assumption that $F^{\prime}(\boldsymbol{k}, u)$ vanishes only at isolated values of $u$.

The solutions (5.3) are normalized such that integration over $\boldsymbol{v}$ yields unity. There are however also solutions of (5.2) with vanishing integral, which therefore do not give rise to a charge density. The dependence on $v_{z}$ of these solutions must be given by a delta-function, whereas the dependence on $v_{x}$ and $v_{y}$ must be such that the integral over $\boldsymbol{v}$ vanishes. We shall consider a series of solutions

$$
g_{\boldsymbol{k}, u}^{v}(\boldsymbol{v})=\frac{\partial f_{0} / \partial v_{z}}{F^{\prime}\left(\boldsymbol{k}, v_{z}\right)} P_{\boldsymbol{k}, u}^{v}\left(v_{x}, v_{y}\right) \delta\left(u-v_{z}\right), \quad(\boldsymbol{v}=1,2, \ldots)
$$

where $P_{k, u}^{v}\left(v_{x}, v_{y}\right)$ is a polynomial in $v_{x}$ and $v_{y}$ with

$$
\int \frac{\partial f_{0} / \partial v_{z}}{F^{\prime}\left(\boldsymbol{k}, v_{z}\right)} P_{\boldsymbol{k}, u}^{v}\left(v_{x}, v_{y}\right) \delta\left(u-v_{z}\right) \mathrm{d} \boldsymbol{v}=0 .
$$

Moreover, the polynomials are so chosen that the following orthonormality relations hold

$$
\int \frac{\partial f_{0} / \partial v_{z}}{F^{\prime}\left(\boldsymbol{k}, v_{z}\right)} P_{\boldsymbol{k}, u}^{\mu}\left(v_{x}, v_{y}\right) P_{\boldsymbol{k}, u}^{v}\left(v_{x}, v_{y}\right) \delta\left(u-v_{z}\right) d v=\delta_{\mu \nu} .
$$

If one extends the series of polynomials so that (5.6) also holds for $P_{\boldsymbol{k}, u}^{0}(\boldsymbol{v})=$ $=1$, which is possible on account of (5.5), then the series can be constructed in a unique fashion from (5.6) by successively including higher powers of $v_{x}$ and $v_{y}$. In section 8 this procedure will be carried out explicitly for the case where $f_{0}(\boldsymbol{v})$ is the Maxwell-Boltzmann distribution.

It is clear that the solutions (5.3) and (5.4) cannot serve for expansion purposes in regions of $\boldsymbol{v}$-space where $\partial f_{0} / \partial v_{z}$ vanishes identically. We shall make the simplifying assumption that $\partial f_{0} / \partial v_{z}$ vanishes only at isolated points. It is possible to avoid the assumptions we have made in the present section but then matters would be more complicated mathematically, whereas the theory would not gain in physical content. Moreover, in the Maxwell-Boltzmann case which is of most interest to us the assumptions are clearly satisfied. 
6. Adjoint solutions. It will be convenient to consider also solutions of the equation adjoint to (5.2), which reads

$$
\left(u-v_{z}\right) \tilde{g}_{k, u}(\boldsymbol{v})+u_{p}^{2} \int \frac{\partial f_{0}}{\partial v_{z}^{\prime}} \tilde{g}_{k, u}\left(\boldsymbol{v}^{\prime}\right) \mathrm{d} \boldsymbol{v}^{\prime}=0 .
$$

The solutions of this equation are of two types: a) solutions for which the integral in (6.1) differs from zero, b) solutions for which the integral vanishes. These two cases will be considered separately.

a) Solutions of this type, which are denoted by an upper index zero, can be normalized such that

$$
-u_{p}^{2} \int \frac{\partial f_{0}}{\partial v_{z}} \widetilde{g}_{k, u}^{0}(\boldsymbol{v}) \mathrm{d} \boldsymbol{v}=N(\boldsymbol{k}, u),
$$

where $N(\boldsymbol{k}, u)$ can still be chosen. Inserting in (6.1) one finds that the solutions are

$$
\tilde{g}_{\boldsymbol{k}, u}^{0}(\boldsymbol{v})=P \frac{N(\boldsymbol{k}, u)}{u-v_{z}}+M(\boldsymbol{k}, u) \delta\left(u-v_{z}\right),
$$

where $M(\boldsymbol{k}, u)$ follows from the normalization (6.2),

$$
M(\boldsymbol{k}, u)=\pi X(\boldsymbol{k}, u) N(\boldsymbol{k}, u) / Y(\boldsymbol{k}, u)
$$

It is convenient to take

$$
N(\boldsymbol{k}, u)=Y(\boldsymbol{k}, u) / \pi|Z(\boldsymbol{k}, u)|^{2},
$$

so that

$$
\begin{aligned}
\widetilde{g}_{\boldsymbol{k}, u}^{0}(\boldsymbol{v}) & =\frac{1}{|Z(\boldsymbol{k}, u)|^{2}}\left[\frac{P}{\pi} \frac{Y(\boldsymbol{k}, u)}{u-v_{z}}+X(\boldsymbol{k}, u) \delta\left(u-v_{z}\right)\right]= \\
& =\frac{\delta_{+}\left(u-v_{z}\right)}{Z(\boldsymbol{k}, u)}+\frac{\delta_{-}\left(u-v_{z}\right)}{Z^{*}(\boldsymbol{k}, u)} .
\end{aligned}
$$

From the adjointness of (5.2) and (6.1) it follows that $g_{\boldsymbol{k}, u}^{0}(\boldsymbol{v})$ and $\tilde{g}_{\boldsymbol{k}, u^{\prime}}^{0}(\boldsymbol{v})$ are orthogonal when $u$ and $u^{\prime}$ differ. With the normalization (6.5) one has furthermore

$$
\int g_{k, u}^{0}(v) \tilde{g}_{k, u^{\prime}}^{0}(v) \mathrm{d} v=\delta\left(u-u^{\prime}\right)
$$

This result is aequivalent to van Kampen's solution of the singular integral equation ${ }^{14}$ ); it can also be verified by direct calculation. The solutions (4.9) and (6.6) correspond to class la) as defined by Case ${ }^{15}$ ). The classes 1 b) and $1 c$ ) do not occur due to our first assumption of the previous section. We finally remark that solutions (6.6) for which $Y(\boldsymbol{k}, u)$ and therefore $F^{\prime}(\boldsymbol{k}, u)$ accidentally vanish are strictly speaking of type b) as defined above. 
b) It follows from (6.1) that for solutions of this type the dependence on $v_{z}$ must be given by a delta-function. As regards the dependence on $v_{x}$ and $v_{y}$ one has a free choice as long as the integral in (6.1) vanishes. Comparing with (5.5) one finds the series of solutions

$$
\tilde{g}_{k, u}^{v}(v)=P_{k, u}^{v}(v) \delta\left(u-v_{z}\right) \quad(v=1,2, \ldots) .
$$

With the aid of (5.6) this yields the orthonormality relation

$$
\int g_{k, u}^{\mu}(v) \tilde{g}_{k, u^{\prime}}^{v}(v) \mathrm{d} v=\delta_{\mu \nu} \delta\left(u-u^{\prime}\right)
$$

This relation also holds for $\mu$ or $v=0$.

7. Expansion in normal mode solutions. A linear superposition of normal mode solutions of the form

$$
f_{\boldsymbol{k}}(\boldsymbol{v}, t)=\sum_{\boldsymbol{v}} \int C_{\boldsymbol{v}}(\boldsymbol{k}, u) g_{\boldsymbol{k}, u}^{v}(\boldsymbol{v}) \mathrm{e}^{-i k u t} \mathrm{~d} u
$$

will again be a solution of equation (4.3). If the normal mode solutions constitute a complete set, then any solution of (4.3) can be written in this form. We shall not endeavour to prove completeness because a rigorous proof would involve conditions on the function $f_{0}(v)$ which have little to do with physics. Rather, we shall assume completeness. It will be shown in the next section that in the Maxwell-Boltzmann case the completeness proof is trivial.

The set of normal mode solutions has yet to be extended to include the case $\boldsymbol{k}=0$, which has not been considered until now. Again one has a certain freedom. One can, for example, prefer a direction in $\boldsymbol{k}$-space and take as the basic set in which to expand the $\boldsymbol{k}=0$ component of the distribution function

$$
g_{\boldsymbol{k}, u}^{\nu}(\boldsymbol{v})=\frac{\partial f_{0} / \partial v_{z}}{F^{\prime}\left(\boldsymbol{k}, v_{z}\right)} P_{\boldsymbol{k}, u}^{v}\left(v_{x}, v_{y}\right) \delta\left(u-v_{z}\right) \quad(v=0,1,2, \ldots) .
$$

These functions in fact depend on the direction of $\boldsymbol{k}$ only. The restriction $\int f_{1}(\boldsymbol{r}, \boldsymbol{v}) \mathrm{d} \boldsymbol{r} \mathrm{d} \boldsymbol{v}=0$ implies however that the $\boldsymbol{k}=0$ component of the distribution function vanishes when integrated over $v$; therefore the term $v=0$ of the series (7.2) will always be absent in an expansion of this component.

Finally the following expansion theorem can be stated: Any perturbed distribution function $f_{1}(\boldsymbol{r}, v)$ can be written

$$
f_{1}(\boldsymbol{r}, \boldsymbol{v})=\frac{\Omega}{8 \pi^{3}} \sum_{\boldsymbol{k}, \boldsymbol{v}}^{\prime} \int \mathrm{d} u C_{\nu}(\boldsymbol{k}, u) g_{\boldsymbol{k}, u}^{v}(\boldsymbol{v}) \mathrm{e}^{i \boldsymbol{k} \cdot \boldsymbol{r}},
$$

where the coefficients $C_{v}(k, u)$ are given by

$$
C_{\nu}(\boldsymbol{k}, u)=\frac{1}{8 \pi^{3}} \int \tilde{g}_{\boldsymbol{k}, u}^{\nu}(\boldsymbol{v}) \mathrm{e}^{-i \boldsymbol{k} \cdot \boldsymbol{r}} f_{1}(\boldsymbol{r}, \boldsymbol{v}) \mathrm{d} \boldsymbol{r} \mathrm{d} \boldsymbol{v} ;
$$


the prime on the summation sign in (7.3) indicates that the term $\boldsymbol{k}=0$, $v=0$ is absent. Furthermore, the solution of the linearized Vlasov equation (4.1) with given initial distribution (7.3) at $t=0$ is obtained by multiplying every coefficient $C_{\boldsymbol{v}}(\boldsymbol{k}, u)$ by a harmonic factor $\exp (-i k u t)$.

8. The Maxwell-Boltzmann case. In this section explicit expressions will be given for the normal mode solutions and their adjoints for the case that $f_{0}(v)$ is the Maxwell-Boltzmann distribution (3.4). One readily sees that in this case the normal mode solutions of section 5 are

$$
\begin{aligned}
& g_{\boldsymbol{k}, u}^{v}(\boldsymbol{v})=\frac{1}{2 \pi v_{t h}^{2}} \exp \left[-\frac{v_{x}^{2}+v_{y}^{2}}{2 v_{t h}^{2}}\right]\left[\frac{P}{\pi} \frac{Y_{M B}\left(\boldsymbol{k}, v_{z}\right)}{u-v_{z}}+X_{M B}(\boldsymbol{k}, u) \delta\left(u-v_{z}\right)\right], \\
& g_{\boldsymbol{k}, u}^{v}(\boldsymbol{v})=\frac{1}{2 \pi v_{t h}^{2}} \exp \left[-\frac{v_{x}^{2}+v_{u t}^{2}}{2 v_{t h}^{2}}\right] \frac{H_{m}\left(v_{x} / v_{t h} \sqrt{ } 2\right) H_{n}\left(v_{y} / v_{t h} \sqrt{ }\right)}{\sqrt{2^{m} m ! 2^{n} n !}}-\delta\left(u-v_{z}\right), \\
& (v>0)
\end{aligned}
$$

where $v_{t h}=(\beta m)^{-\frac{1}{2}}$ and where $v$ stands for the pair $(m, n)=(1,0),(0,1)$, $(2,0),(1,1),(0,2)$, etc.. The functions $H_{n}$ are the usual Hermite-polynomials. The adjoint solutions of section 6 are given by

$$
\begin{aligned}
\tilde{g}_{\boldsymbol{k}, u}^{u}(\boldsymbol{v}) & =\frac{1}{\left|Z_{M B}(\boldsymbol{k}, u)\right|^{2}}\left[\frac{P}{\pi} \frac{Y_{M B}(\boldsymbol{k}, u)}{u-v_{z}}+X_{M B}(\boldsymbol{k}, u) \delta\left(u-v_{z}\right)\right], \\
\tilde{g}_{\boldsymbol{k}, u}^{\prime \prime}(\boldsymbol{v}) & =\frac{H_{m}\left(v_{x} / v_{t h} \sqrt{ } 2\right) H_{n}\left(v_{y} / v_{t h} \sqrt{ } 2\right)}{\sqrt{2^{m} m ! 2^{n} n !}} \delta\left(u-i_{z}^{\prime}\right), \quad(v>0) .
\end{aligned}
$$

The solutions (8.1) constitute a complete set into which any function $f(\boldsymbol{v})$ can be expanded. First one integrates over $v x$ and $v_{y}$ and determines the coefficients $C_{0}(\boldsymbol{k}, u)$; completeness of the functions $g_{\boldsymbol{k}, u}^{\prime \prime}(\boldsymbol{v})$ integrated over $v_{x}$ and $v_{y}$ has been proved by van Kam pen $\left.{ }^{14}\right)$. The remainder of $f(\boldsymbol{v})$ when the superposition of functions $g_{\boldsymbol{k}, u}^{0}(\boldsymbol{v})$ with coefficients $C_{0}(\boldsymbol{k}, u)$ is subtracted belongs to a space of functions in which the $g_{\boldsymbol{k}, u}^{\boldsymbol{v}}(\boldsymbol{v})$ with $v>0$ evidently constitute a complete set; this follows from the completeness of the Hermitefunctions in one dimension.

9. Probability distribution of the coefficients $C_{v}(\boldsymbol{k}, u)$. The probability ensemble (3.7) of 1-particle distribution functions can now be transformed into a probability distribution for the coefficients $C_{\boldsymbol{\nu}}(\boldsymbol{k}, u)$. If the expansion (7.3) is inserted into (3.7) one obtains

$$
\begin{aligned}
& P\left\{j_{1}(\boldsymbol{r}, \boldsymbol{v})\right\}= \\
& =\exp \left[-\frac{\left(8 \pi^{3}\right)^{2}}{\Omega} \sum_{\boldsymbol{k}, v, v^{\prime}}^{\prime} \int \mathrm{d} \boldsymbol{v} \mathrm{d} u \mathrm{~d} u^{\prime} C_{\boldsymbol{v}}(\boldsymbol{k}, u) C_{v^{\prime}}^{*}\left(\boldsymbol{k}, u^{\prime}\right) \frac{g_{\boldsymbol{k}, u}^{v^{\prime}}(\boldsymbol{v}) g_{\boldsymbol{k}, u^{\prime}}^{\nu^{\prime}}(\boldsymbol{v})}{2 n_{0} f_{M B}(\boldsymbol{v})}-\right. \\
& \left.-\frac{\left(8 \pi^{3}\right)^{2}}{\Omega} \sum_{\boldsymbol{k}}^{\prime} \frac{2 \pi \beta e^{2}}{k^{2}} \int \mathrm{d} u \mathrm{~d} u^{\prime} C_{0}(\boldsymbol{k}, u) C_{0}^{*}\left(\boldsymbol{k}, u^{\prime}\right)\right] / \int P .
\end{aligned}
$$


Use has been made of the fact that the $g_{k, u}^{v}(v)$ are real and that integration over velocity yields unity for $\nu=0$ and zero otherwise. Some care must be taken in performing the integration over $v$ in the first term of the exponent in (9.1). The order of integration over $v$ and $u$ and $u^{\prime}$ may be permuted provided one uses the symbolic relation

$$
\begin{array}{r}
P \int \frac{f(y)}{(x-y)\left(x^{\prime}-y\right)} \mathrm{d} y=\frac{P}{x-x^{\prime}} \int f(y)\left[\frac{1}{y-x}-\frac{1}{y-x^{\prime}}\right] \mathrm{d} y+ \\
+\pi^{2} f(x) \delta\left(x-x^{\prime}\right) .
\end{array}
$$

This is aequivalent to the Poincare-Bertrand transformation formula. With the normal mode solutions (8.1) one finds for the $v=v^{\prime}=0$ term using (9.2)

$$
\int \frac{g_{k, u}^{0}(\boldsymbol{v}) g_{k, u^{\prime}}^{0}(\boldsymbol{v})}{f_{M B}(\boldsymbol{v})} \mathrm{d} \boldsymbol{v}=\frac{\left|Z_{M B}(\boldsymbol{k}, u)\right|^{2}}{F_{M B}(u)} \delta\left(u-u^{\prime}\right)-\frac{4 \pi n_{0} e^{2} \beta}{k^{2}}
$$

For the other terms one finds

$$
\int \frac{g_{\boldsymbol{k}, u}^{v}(\boldsymbol{v}) g_{\boldsymbol{k}, u^{\prime}}^{v^{\prime}}(\boldsymbol{v})}{f_{\boldsymbol{M} B}(\boldsymbol{v})} \mathrm{d} \boldsymbol{v}=\frac{1}{F_{M B}(u)} \delta\left(u-u^{\prime}\right) \delta_{v, v^{\prime},} \quad\left(v, v^{\prime}\right) \neq(0,0) .
$$

The term $4 \pi n_{0} e^{2} \beta / k^{2}$ in (9.3) cancels the second term in the exponent of (9.1). Inserting (9.3) and (9.4) into (9.1) one finally obtains

$$
P\left\{C_{\nu}(\boldsymbol{k}, u)\right\}=\exp \left[-\frac{8 \pi^{3}}{\Omega} \sum_{\boldsymbol{k}, \nu}^{\prime} \int \mathrm{d} u \frac{\left|C_{\nu}(\boldsymbol{k}, u)\right|^{2}}{2 \Delta_{\nu}(\boldsymbol{k}, u)}\right] / \int P
$$

where

$$
\begin{aligned}
& \Delta_{0}(k, u)=\frac{n_{0}}{8 \pi^{3}} \frac{F_{M B}(u)}{\left|Z_{M B}(k, u)\right|^{2}}, \\
& \Delta_{\nu}(k, u)=\frac{n_{0}}{8 \pi^{3}} F_{M B}(u), \quad(v \neq 0) .
\end{aligned}
$$

The prime on the summation sign indicates that the term $\boldsymbol{k}=0, \boldsymbol{v}=0$ is absent.

The probability distribution of normal mode coefficients (9.5) is obviously stationary since the coefficients depend on time according to

$$
C_{\nu}(\boldsymbol{k}, u, t)=C_{\nu}(\boldsymbol{k}, u, 0) \mathrm{e}^{-i k u t} .
$$

The great advantage of (9.5) over (3.7) is that the exponent in (9.5) is diagonal, which enables one to explicitly calculate probability distributions of quantities which can be expressed in terms of the normal mode coefficients.

10. Joint probability distributions. In this section a general scheme is presented for the calculation of the joint probability distribution of any number of quantities depending linearly on $f_{1}(\boldsymbol{r}, \boldsymbol{v})$ and taken at different 
times. Any such quantity can be expressed linearly in terms of normal mode coefficients at a fixed time, say $t=0$, so that generally it can be written

$$
Q(\boldsymbol{r}, t)=\frac{8 \pi^{3}}{\Omega} \sum_{\boldsymbol{k}, v}^{\prime} \int \mathrm{d} u q_{v}(\boldsymbol{k}, u, \boldsymbol{r}, t) C_{\nu}(\boldsymbol{k}, u, 0) .
$$

In particular, we shall encounter (real) quantities of the form

$$
Q(\boldsymbol{r}, t)=\int \varphi\left(\boldsymbol{r}-\boldsymbol{r}^{\prime}, \boldsymbol{v}^{\prime}\right) f_{1}\left(\boldsymbol{r}^{\prime}, \boldsymbol{v}^{\prime}, t\right) \mathrm{d} \boldsymbol{r}^{\prime} \mathrm{d} \boldsymbol{v}^{\prime} .
$$

In terms of the coefficients $C_{\nu}(\boldsymbol{k}, u, 0)$ this becomes

$$
Q(\boldsymbol{r}, t)=\frac{8 \pi^{3}}{\Omega}-\sum_{\boldsymbol{k}, v}^{\prime} \int \mathrm{d} u a_{\nu}(\boldsymbol{k}, u) \mathrm{e}^{i(\boldsymbol{k} \cdot \boldsymbol{r}-k u t)} C_{\nu}(\boldsymbol{k}, u, 0)
$$

where

$$
a_{\nu}(\boldsymbol{k}, u)=\int \varphi(\boldsymbol{r}, \boldsymbol{v}) g_{\boldsymbol{k}, u}^{v}(\boldsymbol{v}) \mathrm{e}^{-i \boldsymbol{k} \cdot \boldsymbol{r}} \mathrm{d} \boldsymbol{r} \mathrm{d} \boldsymbol{v} .
$$

The probability that the quantity $Q(\boldsymbol{r}, t)$ assumes the value $Q$ one obtains by using the representation

$$
\delta(Q-Q(\boldsymbol{r}, t))=\frac{1}{2 \pi} \int \mathrm{d} \xi \exp [i \xi(Q-Q(\boldsymbol{r}, t))],
$$

averaging the exponential over the ensemble (9.5) and finally performing the integration over $\xi$. Care should be taken to first separate $C_{\nu}(\boldsymbol{k}, u)$ into real and imaginary parts, both of which can independently assume all values between minus and plus infinity. The integral over the continuous variable $u$ causes no difficulty; formally one can first discretize $u$ and afterwards go to the continuous limit again. One only has to deal with a product of Gaussian integrals and the result is

$$
P(Q)=\left(2 \pi \sigma^{2}\right)^{-\frac{1}{2}} \exp \left[-Q^{2} / 2 \sigma^{2}\right],
$$

where

$$
\sigma^{2}=\left\langle Q^{2}(\boldsymbol{r}, t)\right\rangle=\frac{8 \pi^{3}}{\Omega} \sum_{\boldsymbol{k}, \nu}^{\prime} \int\left|q_{\nu}(\boldsymbol{k}, u, \boldsymbol{r}, \iota)\right|^{2} \Delta_{\nu}(\boldsymbol{k}, u) \mathrm{d} u .
$$

For quantities of the form (10.2) $\sigma^{2}$ does not depend on $\boldsymbol{r}$ or $t$.

The joint probability distribution of two quantities $Q_{1}(\boldsymbol{r}, t)$ and $Q_{2}\left(\boldsymbol{r}^{\prime}, t^{\prime}\right)$ is calculated in similar fashion from the average of a product of deltafunctions in the representation (10.5). The final result is

$$
\begin{aligned}
P\left(Q_{1}, Q_{2}\right)=\frac{1}{2 \pi \sigma_{1} \sigma_{2} \sqrt{1-\rho^{2}}} \exp \left\{\left[-\frac{Q_{1}^{2}}{2 \sigma_{1}^{2}}+\right.\right. & \frac{\rho}{\sigma_{1} \sigma_{2}} Q_{1} Q_{2}- \\
& \left.\left.-\frac{Q_{2}^{2}}{2 \sigma_{2}^{2}}\right] /\left(1-\rho^{2}\right)\right\},
\end{aligned}
$$


where $\sigma_{1}^{2}$ and $\sigma_{2}^{2}$ are given by (10.7) and

$$
\begin{aligned}
\rho \sigma_{1} \sigma_{2}=\langle & \left\langle Q_{1}(\boldsymbol{r}, t) Q_{2}\left(\boldsymbol{r}^{\prime}, t^{\prime}\right)\right\rangle= \\
& =\frac{8 \pi^{3}}{\Omega} \sum_{\boldsymbol{k}, \nu}^{\prime} \int \operatorname{Re} q_{1 \nu}(\boldsymbol{k}, u, \boldsymbol{r}, t) q_{2 \nu}^{*}\left(\boldsymbol{k}, u, \boldsymbol{r}^{\prime}, t^{\prime}\right) \Delta_{v}(\boldsymbol{k}, u) \mathrm{d} u .
\end{aligned}
$$

If $Q_{1}$ and $Q_{2}$ are of the form (10.2) one obtains

$$
\begin{aligned}
\rho \sigma_{1} \sigma_{2} & =\left\langle Q_{1}(\boldsymbol{r}, t) Q_{2}\left(\boldsymbol{r}^{\prime}, t^{\prime}\right)\right\rangle= \\
& =\frac{8 \pi^{3}}{\Omega} \sum_{\boldsymbol{k}, v}^{\prime} \int \operatorname{Re} a_{1 v}(\boldsymbol{k}, u) a_{2 v}^{*}(\boldsymbol{k}, u) \mathrm{e}^{i \boldsymbol{k} \cdot\left(\boldsymbol{r}-\boldsymbol{r}^{\prime}\right)-i k u\left(t-t^{\prime}\right)} \Delta_{\nu}(\boldsymbol{k}, u) \mathrm{d} u,
\end{aligned}
$$

which depends only on the differences $\boldsymbol{r}-\boldsymbol{r}^{\prime}$ and $t-t^{\prime}$.

The joint probability distribution of an arbitrary number of quantities is obtained in the same way. Again one finds a Gaussian distribution with dispersions given by (10.7) and correlations between any pair of quantities given by (10.9).

11. Density fluctuations. As a first example of the general results of the previous section we consider fluctuations in the density. The total density is $n(\boldsymbol{r})=n_{0}+n_{1}(\boldsymbol{r})$ and according to (10.2) and (10.4) the density fluctuation $n_{1}(\boldsymbol{r})$ is connected with

$$
\varphi\left(\boldsymbol{r}-\boldsymbol{r}^{\prime}, \boldsymbol{v}^{\prime}\right)=\delta\left(\boldsymbol{r}-\boldsymbol{r}^{\prime}\right), \quad a_{\nu}(\boldsymbol{k}, u)=\delta_{\boldsymbol{v}, \mathbf{0}} .
$$

Equation (10.10) now yields immediately

$$
\left\langle n_{1}(0,0) n_{1}(\boldsymbol{r}, t)\right\rangle=\frac{n_{0}}{\Omega} \sum_{\boldsymbol{k}}^{\prime} \int \operatorname{Re} \frac{F_{M B}(u)}{\left|Z_{M B}(\boldsymbol{k}, u)\right|^{2}} \mathrm{e}^{i(\boldsymbol{k} \cdot \boldsymbol{r}-k u t)} \mathrm{d} u .
$$

In particular

$$
\begin{aligned}
& \left\langle n_{1}(0,0) n_{1}(\boldsymbol{r}, 0)\right\rangle-\frac{n_{0}}{\Omega} \sum_{\boldsymbol{k}}^{\prime} \int \operatorname{Re} \frac{F_{M B}(u)}{\left|Z_{M B}(\boldsymbol{k}, u)\right|^{2}} \mathrm{e}^{i \boldsymbol{k} \cdot \boldsymbol{r}} \mathrm{d} u= \\
& -\frac{n_{0}}{8 \pi^{3}} \int \frac{k^{2}}{k^{2}+\kappa^{2}} \mathrm{e}^{i \boldsymbol{k} \cdot \boldsymbol{r}} \mathrm{d} \boldsymbol{k}=n_{0} \delta(\boldsymbol{r})-\frac{n_{0} \kappa^{2}}{4 \pi} \frac{\mathrm{e}^{-\kappa r}}{r},
\end{aligned}
$$

where $\kappa^{2}=4 \pi n_{0} e^{2} \beta$ is the square inverse of the Debije-length; the integral over $u$ is performed by contour integration.

One can also find the joint probability for a density $n_{0}+n_{1}(\boldsymbol{r})$ in all points of space simultaneously. This is most easily expressed in terms of the Fourier components $n_{k}$ defined by

$$
n_{1}(\boldsymbol{r})=\frac{8 \pi^{3}}{\Omega} \sum_{k}^{\prime} n_{k} \mathrm{e}^{i k \cdot r},
$$

so that at $t=0$

$$
n_{k}=\int C_{0}(\boldsymbol{k}, u, 0) \mathrm{d} u
$$


Hence one finds with (10.1) for the real and imaginary parts of $n_{k}=n_{k}^{\prime}+i n_{k}^{\prime \prime}$, $\begin{array}{ll}\text { for } n_{\boldsymbol{k}}^{\prime} & q_{\nu}\left(\boldsymbol{k}^{\prime}, u\right)-\frac{\Omega}{8 \pi^{3}} \delta_{\nu, 0}\left[\delta_{\boldsymbol{k}^{\prime}, k}+\delta_{\boldsymbol{k}^{\prime},-\boldsymbol{k}}\right] / 2 \\ \text { for } n_{\boldsymbol{k}}^{\prime \prime} & q_{\nu}\left(\boldsymbol{k}^{\prime}, u\right)-\frac{\Omega}{8 \pi^{3}} \delta_{\nu, 0}\left[\delta_{\boldsymbol{k}^{\prime}, k}-\delta_{\boldsymbol{k}^{\prime},-\boldsymbol{k}}\right] / 2 i .\end{array}$

With (10.7) and (10.9) this yields the joint probability distribution

$$
P\left\{n_{1}(\boldsymbol{r})\right\}=\exp \left[-\frac{8 \pi^{3}}{\Omega} \sum_{k}^{\prime} \frac{8 \pi^{3}}{n_{0}}\left(1+\kappa^{2} / k^{2}\right) n_{k}{ }^{2}\right] / \int P
$$

This well-known result ${ }^{11}$ ) can be used to calculate joint probability distributions of quantities which can be linearly expressed in $n_{1}(\boldsymbol{r})$, such as the potential or the electric field, but only for points taken at the same time.

12. Potential and electric field. The electrostatic potential is expressed in normal mode coefficients via

$$
\varphi\left(\boldsymbol{r}-\boldsymbol{r}^{\prime}, \boldsymbol{v}^{\prime}\right)=\frac{e}{\boldsymbol{r}-\boldsymbol{r}^{\prime} !}, \quad a_{\boldsymbol{v}}(\boldsymbol{k}, u) \cdots \frac{4 \pi e}{k^{2}} \delta_{v, \mathbf{0}} .
$$

Hence one finds with (10.9)

$$
\begin{aligned}
\langle\Phi(0,0) \Phi(\boldsymbol{r}, t)\rangle & = \\
& =\frac{n_{0}}{\Omega} \sum_{\boldsymbol{k}}^{\prime} \frac{16 \pi^{2} e^{2}}{k^{4}} \int \frac{F_{M B}(u)}{\left|Z_{M B}(\boldsymbol{k}, u)\right|^{2}} \cos (\boldsymbol{k} \cdot \boldsymbol{r}-k u t) \mathrm{d} u .
\end{aligned}
$$

In particular

$$
\langle\Phi(0,0) \Phi(\boldsymbol{r}, 0)\rangle=\frac{2 n_{0} e^{2}}{\pi} \int \frac{\cos \boldsymbol{k} \cdot \boldsymbol{r}}{k^{2}\left(k^{2}+\kappa^{2}\right)} \cdot \mathrm{d} \boldsymbol{k}=-\begin{gathered}
1-e^{-\boldsymbol{r} \boldsymbol{r}} \\
\beta r
\end{gathered} .
$$

This yields with $(10.6)$

$$
P(\Phi)=(\beta / 2 \pi \kappa)^{\frac{1}{2}} \exp \left[-\beta \Phi^{2} / 2 \kappa\right] .
$$

The electric field is of course intimately connected with the potential. In this case

$$
a_{v}(\boldsymbol{k}, u)=-\frac{4 \pi i e \boldsymbol{k}}{k^{2}} \delta_{v, 0}
$$

Hence

$$
\begin{aligned}
& \left\langle E_{\alpha}(0,0) E_{\beta}(\boldsymbol{r}, t)\right\rangle= \\
& \quad=\frac{16 \pi^{2} n_{0} e^{2}}{\Omega} \underset{\boldsymbol{k}}{\nu^{\prime}} \frac{k_{\alpha} k_{\beta}}{k^{4}} \int \frac{F_{M B}(u)}{\left|Z_{M B}(\boldsymbol{k}, u)\right|^{2}} \cos (\boldsymbol{k} \cdot \boldsymbol{r}-k u t) \mathrm{d} u= \\
& =-\frac{\partial^{2}}{\partial x_{\alpha} \partial x_{\beta}}\langle\Phi(0,0) \Phi(\boldsymbol{r}, t)\rangle .
\end{aligned}
$$


For the average electrostatic energy density one finds

$$
\begin{aligned}
\left\langle\frac{|\boldsymbol{E}(0,0)|^{2}}{8 \pi}\right\rangle & =\frac{2 \pi n_{0} e^{2}}{\Omega} \sum_{k}^{\prime} \int \frac{F_{M B}(u)}{k^{2}\left|Z_{M B}(k, u)\right|^{2}} \mathrm{~d} u= \\
& =\frac{k_{B} T / 2}{\Omega} \sum_{k} \int \frac{1}{1+k^{2} / \kappa^{2}} .
\end{aligned}
$$

The long waves each contribute $k_{B} T / 2$, but the short waves are not sufficiently suppressed so that the total result diverges.

13. Current density fluctuations. The current density is given by

$$
\boldsymbol{j}(\boldsymbol{r}, t)=e \int \boldsymbol{v} f_{1}(\boldsymbol{r}, \boldsymbol{v}, t) \mathrm{d} \boldsymbol{v},
$$

so that in this case

$$
\varphi\left(\boldsymbol{r}-\boldsymbol{r}^{\prime}, \boldsymbol{v}\right)=e \boldsymbol{v} \delta\left(\boldsymbol{r}-\boldsymbol{r}^{\prime}\right) .
$$

The situation is somewhat more complicated than before because now higher normal mode coefficients enter. Since these are defined with respect to a system of axes $\left(\boldsymbol{e}_{x}, \boldsymbol{e}_{y}, \boldsymbol{e}_{z}=\boldsymbol{k} / k\right)$ which is different for every direction of $\boldsymbol{k}$ one needs the transformation from this $\boldsymbol{k}$-system to a fixed coordinate system with axes $\left(\boldsymbol{e}_{1}, \boldsymbol{e}_{2}, \boldsymbol{e}_{3}\right)$. If the $\boldsymbol{e}_{x}$-axis is chosen to lie in the $(1,2)$-plane, which is always possible, the coordinate transformation is given by

$$
\begin{aligned}
& x_{1}=\frac{-k_{2}}{\sqrt{k_{1}^{2}+k_{2}^{2}}} x-\frac{k_{1} k_{3}}{k \sqrt{k_{1}^{2}+k_{2}^{2}}} y+\frac{k_{1}}{k} z \\
& x_{2}=\frac{k_{1}}{\sqrt{k_{1}^{2}+k_{2}^{2}}} x-\frac{k_{2} k_{3}}{k \sqrt{k_{1}^{2}+k_{2}^{2}}} y+\frac{k_{2}}{k} z \\
& x_{3}=
\end{aligned}
$$

If the normal modes are ordered as indicated in section 8 , one finds from (10.4) and (13.2) for the components of $v$ in the $\boldsymbol{k}$-system

$$
\begin{array}{ll}
v_{x}: & a_{v}(\boldsymbol{k}, u)=v_{t h} \delta_{v, 1} \\
v_{y}: & a_{v}(\boldsymbol{k}, u)=v_{t h} \delta_{v, 2} \\
v_{z}: & a_{v}(\boldsymbol{k}, u)=u \quad \delta_{v, 0} .
\end{array}
$$

This yields with (10.3) and the coordinate transformation (13.3)

$$
\begin{aligned}
j_{1}(\boldsymbol{r}, t)=\frac{8 \pi^{3} e}{\Omega} \sum_{\boldsymbol{k}}^{\prime} \int\left[\frac{-k_{2}}{\sqrt{k_{1}^{2}+k_{2}^{2}}} v_{t h} C_{\mathbf{1}}(\boldsymbol{k}, u)-\right. \\
\left.\quad-\frac{k_{1} k_{3}}{k \sqrt{k_{1}^{2}+k_{2}^{2}}} v_{t h} C_{2}(\boldsymbol{k}, u)+\frac{k_{1}}{k} u C_{0}(\boldsymbol{k}, u)\right] e^{i(\boldsymbol{k} \cdot \boldsymbol{r}-k u t)} \mathrm{d} u
\end{aligned}
$$




$$
\begin{aligned}
j_{2}(\boldsymbol{r}, t)=\frac{8 \pi^{3} e}{\Omega} \sum_{\boldsymbol{k}}^{\prime} \int\left[\frac{k_{1}}{\sqrt{k_{1}^{2}+k_{2}^{2}}} v_{t h} C_{1}(\boldsymbol{k}, u)-\right. \\
-\frac{k_{2} k_{3}}{\left.k \sqrt{k_{1}^{2}+k_{2}^{2}} v_{t h} C_{2}(\boldsymbol{k}, u)+\frac{k_{2}}{k} u C_{0}(\boldsymbol{k}, u)\right] \mathrm{e}^{i(\boldsymbol{k} \cdot \boldsymbol{r}-k u t)} \mathrm{d} u} \\
j_{\mathbf{3}}(\boldsymbol{r}, t)=\frac{8 \pi^{3} e}{\Omega} \sum_{\boldsymbol{k}}^{\prime} \int\left[\frac{\sqrt{k_{1}^{2}+k_{2}^{2}}}{k} v_{t h} C_{2}(\boldsymbol{k}, u)+\frac{k_{3}}{k} u C_{0}(\boldsymbol{k}, u)\right] \mathrm{e}^{i(\boldsymbol{k} \cdot \boldsymbol{r}-k u t)} \mathrm{d} u,
\end{aligned}
$$

where the prime on the summation sign indicates that for $\boldsymbol{k}=0$ the terms in $C_{0}(\boldsymbol{k}, u)$ are absent. With the aid of (10.10) one finally obtains

$$
\begin{aligned}
& \left\langle j_{\alpha}(0,0) j_{\beta}(\boldsymbol{r}, t)\right\rangle=\frac{n_{0} e^{2}}{\Omega} \sum_{\boldsymbol{k}}^{\prime} \int\left[v_{t \boldsymbol{t}^{\prime}} \delta_{\alpha \beta}+\right. \\
& \left.\quad+\left(\frac{u^{2}}{\left|Z_{\boldsymbol{M} B}(\boldsymbol{k}, u)\right|^{2}}-v_{t h}^{2}\right) \frac{k_{\alpha} k_{\beta}}{k^{2}}\right] F_{M B}(u) \cos (\boldsymbol{k} \cdot \boldsymbol{r}-k u t) \mathrm{d} u .
\end{aligned}
$$

In particular for $t=0$

$$
\left\langle j_{\alpha}(0,0) j_{\beta}(r, 0)\right\rangle=n_{0} e^{2} v_{t h}^{2} \delta_{\alpha \beta} \delta(\boldsymbol{r}),
$$

where use has been made of

$$
\int \frac{u^{2} F_{M B}(u)}{\left|Z_{M B}(\boldsymbol{k}, u)\right|^{2}} \mathrm{~d} u=v_{t h}^{2}
$$

14. Pressure fluctuations. As a final example we consider fluctuations in the hydrostatic pressure defined by

$$
p=\frac{1}{3} m \int v^{2} f(\boldsymbol{r}, \boldsymbol{v}, t) \mathrm{d} \boldsymbol{v}=p_{0}+p_{1}(\boldsymbol{r}, t)
$$

where

$$
p_{0}=n_{0} / \beta, p_{1}(\boldsymbol{r}, t)=\frac{1}{3} m \int v^{2} f_{1}(\boldsymbol{r}, \boldsymbol{v}, t) \mathrm{d} \boldsymbol{v} .
$$

In the $\boldsymbol{k}$-system one finds for the different velocity components

$$
\begin{array}{ll}
v_{x}^{2}: & a_{\nu}(\boldsymbol{k}, u)=v_{t h}^{2} \delta_{\nu, 0}+v_{t h}^{2} \sqrt{ } 2 \delta_{\nu, 3} \\
v_{y}^{2}: & a_{\nu}(\boldsymbol{k}, u)=v_{t h}^{2} \delta_{\nu, 0}+v_{t h}^{2} \sqrt{ } 2 \delta_{\nu, 5} \\
v_{z}^{2}: & a_{\nu}(\boldsymbol{k}, u)=\left(u^{2}-u_{p}^{2}\right) \delta_{\nu, 0} .
\end{array}
$$

Since the trace is invariant under the transformation from the $\boldsymbol{k}$-system to the fixed system of coordinates this yields

$$
\begin{aligned}
p_{1}(\boldsymbol{r}, t)=\frac{1}{3} m \frac{8 \pi^{3}}{\Omega} \sum_{\boldsymbol{k}}^{\prime} \int\left[\left(u^{2}-u_{p}^{2}+2 v_{t h}^{2}\right) C_{0}(\boldsymbol{k}, u)+\right. \\
\left.+v_{t h}^{2} \sqrt{ } 2 C_{3}(\boldsymbol{k}, u)+v_{t h}^{2} \sqrt{ } 2 C_{5}(\boldsymbol{k}, u)\right] \mathrm{e}^{i(\boldsymbol{k} \cdot \boldsymbol{r}-k u t)} \mathrm{d} u .
\end{aligned}
$$


The prime on the summation sign indicates that for $\boldsymbol{k}=0$ the terms in $C_{0}(k, u)$ are absent.

With the aid of $(10.10)$ one finds for the pressure correlations

$$
\begin{gathered}
\left\langle p_{1}(0,0) p_{1}(\boldsymbol{r}, t)\right\rangle=\frac{1}{9} m^{2} \frac{n_{0}}{\Omega} \Sigma_{\boldsymbol{k}}^{\prime} \int\left[\left(u^{2}-u_{p}^{2}+2 v_{t h}^{2}\right)^{2} \frac{F_{\boldsymbol{M B}}(u)}{\left|Z_{\boldsymbol{M B}}(\boldsymbol{k}, u)\right|^{2}}+\right. \\
\left.+4 v_{t h}^{4} F_{M B}(u)\right] \cos (\boldsymbol{k} \cdot \boldsymbol{r}-k u t) \mathrm{d} u .
\end{gathered}
$$

In particular for $t=0$

$$
\left\langle p_{1}(0,0) p_{1}(\boldsymbol{r}, 0)\right\rangle=\frac{5}{3} \frac{n_{0}}{\beta^{2}} \delta(\boldsymbol{r})-\frac{n_{0}^{2} e^{2}}{\beta} \frac{\mathrm{e}^{-\kappa r}}{r},
$$

where use has been made of (13.8) and of

$$
\int \frac{u^{4} F_{M B}(u)}{\left|Z_{M B}(\boldsymbol{k}, u)\right|^{2}} \mathrm{~d} u=3 v_{t h}^{4}+u_{p}^{2} v_{t h}^{2} .
$$

The second term in (14.6) is the deviation from the ideal gas result.

15. Response to an external force. In the next two sections it will be investigated in what manner an external force applied to the plasma influences the fluctuations. The ensemble (3.7) which describes the fluctuations when originally the plasma is in equilibrium will be modified by the external force. This perturbation can be calculated within the bounds of linearized Vlasov theory.

For concreteness the external force will be regarded to be caused by an „external" charge distribution

$$
e n_{e x}(\boldsymbol{r}, t)=e \int n_{e x}(\boldsymbol{k}, \omega) \mathrm{e}^{i(\boldsymbol{k} \cdot \boldsymbol{r}-\boldsymbol{m} t)} \mathrm{d} \boldsymbol{k} \mathrm{d} \omega .
$$

The equation of motion for $f_{1}(\boldsymbol{r}, \boldsymbol{v}, t)$ then becomes

$$
\begin{aligned}
\frac{\partial f_{1}}{\partial t}+\boldsymbol{v} \cdot \frac{\partial f_{1}}{\partial \boldsymbol{r}} & +\frac{n_{0} e^{2}}{m} \frac{\partial f_{0}}{\partial \boldsymbol{v}} . \\
& \cdot \int \frac{\boldsymbol{r}-\boldsymbol{r}^{\prime}}{\left|\boldsymbol{r}-\boldsymbol{r}^{\prime}\right|^{3}}\left[\int f_{1}\left(\boldsymbol{r}^{\prime}, \boldsymbol{v}^{\prime}, t\right) \mathrm{d} \boldsymbol{v}^{\prime}+n_{e x}\left(\boldsymbol{r}^{\prime}, t\right)\right] \mathrm{d} \boldsymbol{r}^{\prime}=0 .
\end{aligned}
$$

Multiplying this equation by $\tilde{g}_{\boldsymbol{k}, u}^{v}(\boldsymbol{v}) \exp (-i \boldsymbol{k} \cdot \boldsymbol{r})$ and integrating over $\boldsymbol{r}$ and $\boldsymbol{v}$ one finds that the corresponding equation of motion for the normal mode coefficients $C_{0}(\boldsymbol{k}, u)$ is given by

$$
\frac{\partial C_{0}(\boldsymbol{k}, u)}{\partial t}+i k u C_{0}(\boldsymbol{k}, u)+\frac{i k}{\pi} \frac{Y(\boldsymbol{k}, u)}{|Z(\boldsymbol{k}, u)|^{2}} n_{e x}(\boldsymbol{k}, t)=0,
$$

whereas the motion of the other coefficients is still harmonic,

$$
\frac{\partial C_{\nu}(\boldsymbol{k}, u)}{\partial t}+i k u C_{\nu}(\boldsymbol{k}, u)=0 \quad \text { for } \quad v>0 .
$$


The solution of equation (15.3) is

$$
\begin{aligned}
& C_{0}(\boldsymbol{k}, u, t)-\frac{Y(\boldsymbol{k}, u) / \boldsymbol{\pi}}{|Z(\boldsymbol{k}, u)|^{2}} \cdot \\
& \quad \cdot \int \frac{\mathrm{e}^{i k u\left(t_{0}-t\right)-i \omega t_{0}}-\mathrm{e}^{-i \omega t}}{u-\omega / k} \cdot n_{e x}(\boldsymbol{k}, \omega) \mathrm{d} \omega+C_{0}\left(\boldsymbol{k}, u, t_{0}\right) \mathrm{e}^{-i k u\left(t-t_{0}\right)} .
\end{aligned}
$$

Hence one finds that if at $t=-\infty$ the probability distribution is given by the equilibrium ensemble (3.7) or (9.5), then at an arbitrary time $t$ it is given by

$$
\begin{aligned}
& P_{t}\left\{f_{1}(\boldsymbol{r}, \boldsymbol{v})\right\}= \\
& =\exp \left\{-\frac{8 \pi^{3}}{\Omega}-\sum_{\boldsymbol{k}, v} \int\left[\frac{\left|C_{\nu}(\boldsymbol{k}, u)\right|^{2}}{2 \Delta_{\nu}(\boldsymbol{k}, u)}-\operatorname{Re} \gamma^{*}(\boldsymbol{k}, u, t) C_{0}(\boldsymbol{k}, u)\right] \mathrm{du}\right\} / \int P .
\end{aligned}
$$

where

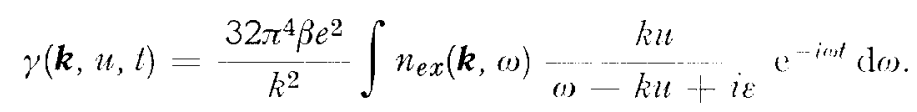

In particular, if the external force does not depend on time, the extra term in (15.6) is precisely $-\beta$ times the interaction energy. For in case of a static extcrnal charge distribution the interaction energy is

$e^{2} \int \frac{f_{1}(\boldsymbol{r}, \boldsymbol{v}) n_{e x}\left(\boldsymbol{r}^{\prime}\right)}{\left|\boldsymbol{r}-\boldsymbol{r}^{\prime}\right|} \mathrm{d} \boldsymbol{r} \mathrm{d} \boldsymbol{v} \mathrm{d} \boldsymbol{r}^{\prime}=\frac{8 \pi^{3}}{\Omega} \sum_{\boldsymbol{k}}^{\prime} \frac{32 \pi^{4} e^{2}}{k^{2}} n_{e \cdot x}^{*}(\boldsymbol{k}) \int C_{0}(\boldsymbol{k}, u) \mathrm{d} u$.

16. Joint probability distributions when an external jorce is present. The joint probability distribution of quantities depending linearly on $f_{1}(\boldsymbol{r}, \boldsymbol{v})$ will also be influenced by an external force. In order to calculate this influence from (15.6) it is convenient to consider this ensemble at a fixed time, say $t=0$, and to express all quantities with the aid of (15.5) in terms of normal mode coefficients taken at $t=0$. That is, one writes (cf. section 10)

$$
\begin{aligned}
& Q(\boldsymbol{r}, t)=\frac{8 \pi^{3}}{\Omega} \sum_{\boldsymbol{k}, v}^{\prime} \int \mathrm{d} u a_{\boldsymbol{v}}(\boldsymbol{k}, u) \mathrm{e}^{i \boldsymbol{k} \cdot \boldsymbol{r}} C_{\boldsymbol{v}}(\boldsymbol{k}, u, t)= \\
& =\frac{8 \pi^{3}}{\Omega} \sum_{\boldsymbol{k}, v}^{\prime} \int \mathrm{d} u\left[a_{\nu}(\boldsymbol{k}, u) \mathrm{e}^{i(\boldsymbol{k} \cdot \boldsymbol{r}-k u t)} C_{\nu}(\boldsymbol{k}, u, 0)+a_{0}(\boldsymbol{k}, u) \mathrm{e}^{i \boldsymbol{k} \cdot \boldsymbol{r}} I(\boldsymbol{k}, u, t)\right],
\end{aligned}
$$

where

$$
I(\boldsymbol{k}, u, t)=\frac{Y(\boldsymbol{k}, u) / \pi}{|Z(\boldsymbol{k}, u)|^{2}} \int \frac{\mathrm{e}^{-i k u t}-\mathrm{e}^{-i \omega t}}{u-\omega / k} n_{e x}(\boldsymbol{k}, \omega) \mathrm{d} \omega .
$$

If one now calculates the joint probability distribution of two quantities $Q_{1}(\boldsymbol{r}, t)$ and $Q_{2}\left(\boldsymbol{r}^{\prime}, t^{\prime}\right)$ by the same procedure as was used in section 10 one 
obtains

$$
\begin{aligned}
& P\left(Q_{1}, Q_{2}\right)=\frac{1}{2 \pi \sigma_{1} \sigma_{2} \sqrt{1-\rho^{2}}} \exp \left\{\left[-\frac{\left(Q_{1}-\left\langle Q_{1}\right\rangle\right)^{2}}{2 \sigma_{1}^{2}}+\right.\right. \\
& \left.\left.+\frac{\rho}{\sigma_{1} \sigma_{2}}\left(Q_{1}-\left\langle Q_{1}\right\rangle\right)\left(Q_{2}-\left\langle Q_{2}\right\rangle\right)-\frac{\left(Q_{2}-\left\langle Q_{2}\right\rangle\right)^{2}}{2 \sigma_{2}^{2}}\right] /\left(1-\rho^{2}\right)\right\},
\end{aligned}
$$

where

$$
\begin{gathered}
\sigma^{2}=\left\langle(Q-\langle Q\rangle)^{2}\right\rangle=\frac{8 \pi^{3}}{\Omega} \sum_{\boldsymbol{k}, v}^{\prime} \int\left|a_{v}(\boldsymbol{k}, u)\right|^{2} \Delta_{\nu}(\boldsymbol{k}, u) \mathrm{d} u, \\
\rho \sigma_{1} \sigma_{2}=\left\langle\left(Q_{1}-\left\langle Q_{1}\right\rangle\right)\left(Q_{2}-\left\langle Q_{2}\right\rangle\right)\right\rangle= \\
=\frac{8 \pi^{3}}{\Omega} \sum_{\boldsymbol{k}, v}^{\prime} \int \operatorname{Re} a_{1 v}(\boldsymbol{k}, u) a_{2 v}^{*}(\boldsymbol{k}, u) \mathrm{e}^{i \boldsymbol{k} \cdot\left(\boldsymbol{r}-\boldsymbol{r}^{\prime}\right)-i k u\left(t-t^{\prime}\right)} \Delta_{\nu}(\boldsymbol{k}, u) \mathrm{d} u
\end{gathered}
$$

and

$$
\langle Q\rangle=\frac{8 \pi^{3}}{\Omega} \sum_{\boldsymbol{k}}^{\prime} \int \operatorname{Re} a_{0}(\boldsymbol{k}, u) \gamma(\boldsymbol{k}, u, t) \mathrm{e}^{i \boldsymbol{k} \cdot \boldsymbol{r}} \Delta_{\mathbf{0}}(\boldsymbol{k}, u) \mathrm{d} u .
$$

The joint probality distributions are again Gaussian with the same dispersions and covariances as before, only the avcrages now differ from zero. It can be shown that for the average (16.5) one can also write

$$
\begin{aligned}
\langle Q\rangle=\frac{8 \pi^{3}}{\Omega} \sum_{\boldsymbol{k}, v} \int \operatorname{Re} \varphi_{\boldsymbol{k}}(\boldsymbol{v}) \cdot \\
\quad \frac{-\omega_{p}^{2}}{k^{2} Z(\boldsymbol{k}, \omega / k)} \frac{\boldsymbol{k} \cdot \partial f_{0} / \partial \boldsymbol{v}}{\omega-\boldsymbol{k} \cdot \boldsymbol{v}+i_{\varepsilon}} n_{e x}(\boldsymbol{k}, \omega) \mathrm{e}^{i(\boldsymbol{k} \cdot \boldsymbol{r}-\omega t)} \mathrm{d} \boldsymbol{v} \mathrm{d} \omega,
\end{aligned}
$$

where

$$
\varphi_{\boldsymbol{k}}(\boldsymbol{v})=\int \varphi(\boldsymbol{r}, \boldsymbol{v}) \mathrm{e}^{-i \boldsymbol{k} \cdot \boldsymbol{r}} \mathrm{d} \boldsymbol{r} .
$$

This is exactly the result one would find from the linearized Vlasov theory, equation (15.2), which is not surprising because the nonstationary ensemble (15.6) has been established with the aid of this theory.

17. Test particle. As an application of the theory of the preceding two sections one may consider the test particle problem. One of the particles is singled out as test particle and treated as external charge acting on the rest of the plasma. The average potential caused by an external charge distribution is according to (12.1) and (16.5)

$$
\langle\Phi(\boldsymbol{r}, t)\rangle=\frac{8 \pi^{3}}{\Omega} \Sigma_{\boldsymbol{k}}^{\prime} \int \operatorname{Re} \frac{4 \pi e}{k^{2}} n_{e x}(\boldsymbol{k}, \omega)\left[\frac{1}{Z(\boldsymbol{k}, \omega / k)}-1\right] \mathrm{e}^{i(\boldsymbol{k} \cdot \boldsymbol{r}-\omega t)} \mathrm{d} \omega .(17.1)
$$

Hence one obtains by treating a particle of velocity $v_{0}$ as test particle

$$
\langle\Phi(\boldsymbol{r}, t)\rangle_{\boldsymbol{v}_{\mathrm{o}}}=\frac{1}{\Omega} \sum_{\boldsymbol{k}}^{\prime} \frac{4 \pi e}{k^{2}}\left[\frac{1}{Z\left(\boldsymbol{k}, \boldsymbol{k} \cdot \boldsymbol{v}_{0} / k\right)}-1\right] \mathrm{e}^{i \boldsymbol{k} \cdot\left(\boldsymbol{r}-\boldsymbol{r}_{0}-\boldsymbol{v}_{0} t\right)}
$$


For a test particle at rest this becomes

$$
\left\langle\Phi(\boldsymbol{r})_{\mathbf{0}_{0}}--e \frac{1-\exp \left(-\kappa\left|\boldsymbol{r}-\boldsymbol{r}_{0}\right|\right)}{\left|\boldsymbol{r}-\boldsymbol{r}_{0}\right|} .\right.
$$

The probability distribution of the potential at the position of the charge is given by

$$
P_{\epsilon, v_{0}}(\Phi)=(\beta / 2 \pi \kappa)^{?} \exp \left[-\beta\left(\Phi-\langle\Phi\rangle_{\boldsymbol{v}_{0}}\right)^{2} / 2 \kappa\right]
$$

where

$$
\langle\boldsymbol{\Phi}\rangle_{\boldsymbol{v}_{0}}=\frac{1}{\Omega} \Sigma_{\boldsymbol{k}}^{\prime} \frac{4 \pi e}{k^{2}}\left[\frac{1}{Z\left(\boldsymbol{k}, \boldsymbol{k} \cdot \boldsymbol{v}_{0} / k\right)}-1\right] .
$$

If the particle is at rest (17.3) yields

$$
\langle\Phi\rangle_{0}--e \kappa .
$$

The probability distribution (17.4) only agrees with the result of Jackson and Klein (ref. 12, eq. (17)) when the particle is at rest. The present calculation takes the deformation of the polarization cloud into account when the particle is in motion.

18. Discussion of the non-equilibrium case. As was already remarked in the introduction, the results for the density fluctuations, field correlations, etc. obtained from the equilibrium ensemble (3.7) in conjunction with linearized Vlasov theory are identical with those obtained from other theories. In fact, it can be shown that the correlation function $W_{12}^{(1)}\left(\boldsymbol{r}_{1}, \boldsymbol{v}_{1}, t\right.$; $\left.\boldsymbol{r}_{2}^{\prime}, \boldsymbol{v}_{2}^{\prime}, t^{\prime}\right)$ occurring in Rostoker's theory (see ref. 5 , eq. $(1,23)$ ) may be derived from the general expression (10.10).

It would be interesting to cxtend the present theory to the non-equilibrium case. This would mean the establishment of a probability ensemble of normal mode coefficients defined with respect to the homogeneous distribution $f_{0}(v)$ characterizing the non-equilibrium state. This ensemble would then also yield the pair correlation function $W_{12}^{(1)}$ in the same manner as in the equilibrium case and therefore e.g. an expression for the collision term. There does not, however, seem to be an independent way to establish the ensemble in the non-equilibrium case. It is of course possible to derive the probability distribution vice versa from the correlation function. The work of Klimontovich and Silin $\left.{ }^{16}\right)^{17}$ ) is in this spirit.

In this connection it is perhaps of interest to mention that the thermal equilibrium ensemble (3.7) can be obtained in an alternative fashion. In fact, it may be shown that in the random phase approximation of Bohm and Pines the quantities

$$
b_{k, u}^{v} \sim \sum_{i} \hat{g}_{k, u}^{y}\left(\boldsymbol{v}_{i}\right) \mathrm{e}^{-i \boldsymbol{k} \cdot \boldsymbol{r}_{i}}
$$

behave as harmonic uscillator variables. With the normalization of section 
6 an extra factor is necessary which yields the correct Poisson brackets for the variables $b_{k, \boldsymbol{u}}^{\boldsymbol{v}}$ and their complex conjugates. For the harmonic oscillators one may introduce the effective Hamiltonian

$$
H_{e f f}=\sum_{k, v}^{\prime} \int_{0}^{\infty} \omega b_{k, u}^{v^{*}} b_{k, u}^{v} \mathrm{~d} u
$$

Finally one can show that when the variables (18.1) are defined with respect to the Maxwell-Boltzmann distribution, the equilibrium ensemble (3.7) is aequivalent with the canonical ensemble based on the effective Hamiltonian (18.2).

If the latter method is applied to the non-equilibrium case and by way of trial all harmonic oscillators are given the same average energy, the results are in disagreement with those obtained by other methods. It is not surprising that outside equilibrium aequipartition does not work, but it is not obvious what else one should try. The results obtained from other methods, e.g. from independent dressed particles, are

$$
\begin{aligned}
& \left\langle C_{0}(\boldsymbol{k}, u) C_{0}^{\star}\left(\boldsymbol{k}^{\prime}, u^{\prime}\right)\right\rangle=\frac{\Omega n_{0}}{\left(8 \pi^{3}\right)^{2}} \frac{F(\boldsymbol{k}, u)}{|Z(\boldsymbol{k}, u)|^{2}} \delta_{k, \boldsymbol{k}^{\prime}} \delta\left(u-u^{\prime}\right) \\
& \left\langle C_{0}(\boldsymbol{k}, u) C_{v}^{*}\left(\boldsymbol{k}^{\prime}, u^{\prime}\right)\right\rangle=\frac{\Omega n_{0}}{\left(8 \pi^{3}\right)^{2}} \delta_{\boldsymbol{k}, \boldsymbol{k}^{\prime}} \frac{\delta\left(u-u^{\prime}\right)}{Z(\boldsymbol{k}, u)} \int f_{0}(\boldsymbol{v}) P_{\boldsymbol{k}, u}^{v}(\boldsymbol{v}) \delta(u-\boldsymbol{k} \cdot \boldsymbol{v} / k) \mathrm{d} \boldsymbol{v} \\
& \left\langle C_{\mu}(\boldsymbol{k}, u) C_{y}^{*}\left(\boldsymbol{k}^{\prime}, u^{\prime}\right)\right\rangle= \\
& (\nu>0) \\
& =\frac{\Omega n_{0}}{\left(8 \pi^{3}\right)^{2}} \delta_{k, k^{\prime}} \delta\left(u-u^{\prime}\right) \int f_{0}(v) P_{k, u}^{\mu}(v) P_{k, u}^{v}(v) \delta(u-k \cdot v / k) \mathrm{d} v . \\
& (\mu, \nu>0)
\end{aligned}
$$

The probability ensemble itself may be taken as Gaussian with these covariances. It reduces to (9.5) when $f_{0}(v)$ is the Maxwell-Boltzmann distribution. It may be remarked that according to linear Vlasov theory the ensemble derived from (18.3) is stationary. Furthermore it is interesting that the coefficients for different $v$ are no longer uncoupled.

Acknowledgement. The author wishes to thank Professor N. G. van Kampen for his stimulating interest.

Received 5-5-64

\section{REFERENCES}

1) Bowles, K. L., Phys. Rev. Letters 1 (1958) 454.

2) Dougherty, J. P., Farley, D. T., Proc. roy. Soc. A 259 (1960) 79.

3) Salpeter, E. E., Phys. Rev. 120 (1960) 1528. 
4) Fejer, J. A., Can. J. Phys. 38 (1960) 1114.

5) Rostoker, N., Nuclear Fusion 1 (1961) 101.

6) Rosenbluth, M. N., Rostoker, N., Phys. of Hluids 5 (1962) 776.

7) Ichinat u, S., Amuals of Physics $\mathbf{2 0}$ (1962) 78.

8) Thompson, W. B., Hubbard, J., Rev. mod. Phys. 32 (1960) 714.

9) Ron, A., Dawson, J., Oberman, C., Phys. Rev. 1322 (1963) 497.

10) Hubbard, J., Proc. roy. Soc. A æ60 (1961) 114.

11) Pines, 1), Bohm, D., Phys. Rev. 85 (1952) 338.

12) Jackson, J. L., Klein, L. S., Phys. of Fluids 7 (1964) 232.

13) van Kampen, N. G., Phys. Rev. 13s (1964) A 362.

14) van Kanpen, N. G., Physica 21 (1955) 949.

15) Case, K. M., Annals of Physics 7 (1959) 349.

16) Klimontovich, Yu. L., Silin, V. P., Sov. Phys. JliTP I5 (1962) 199.

17) Klimontovich, Yu, L., Silin, V. P. Sov. Phys. Doklady 7 (1963) 698. 